\title{
Human derived feeder fibroblasts for the culture of epithelial cells for clinical use
}

\author{
A R O'Callaghan ${ }^{1}$, L Morgan $^{1}{ }^{*}$,J T Daniels ${ }^{1}$, and *M P Lewis ${ }^{2}$
}

\author{
*Authors contributed equally \\ ${ }^{1}$ Department of Ocular Biology and Therapeutics, UCL Institute of Ophthalmology, University College \\ London, London EC1V 9EL. ${ }^{2}$ National Centre for Sport and Exercise Medicine (NCSEM), EPSRC-MRC \\ Centre for Doctoral Training in Regenerative Medicine, School of Sport, Exercise and Health Sciences, \\ Loughborough University, Leicestershire, LE11 3TU. Email of corresponding author: \\ anna.ocallaghan@ucl.ac.uk
}

\section{Abstract:}

Aim: To investigate human oral mucosal fibroblasts (HOMF) and human limbal fibroblasts (HLF) as alternatives to murine $3 \mathrm{~T} 3$ feeder fibroblasts currently used to support epithelial cell expansion for the treatment of limbal epithelial stem cell deficiency.

Methods: HLF and HOMF were compared to $3 T 3$ for their ability to support the culture of human limbal epithelial cells (HLE) and human oral mucosal epithelial cells.

Results: HOMF, but not HLF, were equivalent to $3 T 3$ in terms of the number of epithelial population doublings achieved. HLE co-cultured with HOMF or 3 T3 had similar expression of corneal and putative stem cell markers.

Conclusion: HOMF are a suitable and safer feeder fibroblast alternative to $3 \mathrm{~T} 3$ for the production of epithelial cells for clinical use.

Keywords: cornea, oral mucosa, limbal epithelial stem cell deficiency, stem cell therapy, limbal fibroblasts, oral mucosal fibroblasts, limbal epithelial cells, oral mucosal epithelial cells.

\section{Introduction}

The corneal surface is maintained and repaired by a population of stem cells thought to reside in the limbus called limbal epithelial stem cells (LESC). Injury or disease can result in a deficiency of these LESC, a condition known as limbal stem cell deficiency (LSCD). In LSCD the cornea becomes opaque as the conjunctival epithelium migrates across the limbus and onto the cornea. This affects vision, and is accompanied by inflammation, vascularisation, and severe discomfort. LSCD has been successfully treated using cultured cell therapies: cultivated limbal epithelial transplantation (CLET) and cultivated oral mucosal epithelial transplantation (COMET).

Multiple protocols for ex-vivo expansion of limbal epithelial cells have been successfully used to treat patients with LSCD (reviewed by Tseng et al. [1]). The starting point for these therapies is a small limbal biopsy taken from the healthy eye of the patient if available (unilateral disease), or from a donor eye for cases where both eyes are affected by LSCD (bilateral disease). One of the 
differences is whether HLE are obtained from the biopsy by explant culture or as a single cell suspension. The majority of these methods utilise 3T3s as feeder cells, either in direct or indirect contact with HLE. In this study epithelial cells were isolated from limbal or oral mucosal tissue as a single cell suspension, and expanded by co-culturing with 3T3, HLF or HOMF feeder fibroblasts i.e. direct contact approach. COMET is used for patients with bilateral LSCD, where a small oral biopsy is taken from the patient from which to culture oral mucosal epithelial cells. An advantage of COMET over using donor HLE is that autologous (patient's own) cells can be used thus eliminating the need for immunosuppression and its associated side effects. For COMET, 3T3s have been used in nearly all clinical studies for ex-vivo expansion of HOME (reviewed by Utheim et al. [2]).

In the limbal stem cell "niche", the microenvironment which controls/regulates stem cell fate, the stem cells are in close contact with niche cells [3]. It has been suggested that 3T3s act as a surrogate niche cell for HLE culture [1]. For HLE isolated from limbal tissue as a single cell suspension, direct contact between 3T3s and HLE has been shown to be important for initiating epithelial colonies on tissue culture plastic [4]. Indirect contact with 3T3s has been shown to be beneficial for delaying epithelial differentiation for explant cultured HLE on denuded amniotic membrane [5]. A combination of direct and indirect contact between $3 T 3$ feeder cells and HLEs has been shown to produce more progenitor cells than direct or indirect contact alone [4].

Safety and efficacy are both important considerations for GMP. The use of a 3T3 feeder layer is currently the gold standard method for culturing and maintaining epithelial stem cells. Although $3 T 3$ s are murine derived cells and cell therapies should ideally be animal product free, this culture method is currently optimal and is approved for clinical use until a suitable alternative can be found. Although there is no evidence to suggest that 3T3s have had a harmful effect for patients, and very few if any 3T3s would be transferred with the epithelial graft to the patient [6], it is important to ensure that cell grafts are as safe as possible. In order to fully eliminate the risk of 3T3s transmitting murine viruses to human cells, these cells would need to be removed from the culture process.

The use of human derived feeder fibroblasts represents an alternative to 3T3s and has been investigated for several fibroblast types [7-11], although these have yet to be used in the clinic. Although there are advantages in using a cell line such as reducing variability between different donor cells, the use of allogeneic (donor) human fibroblasts still carries a risk of disease transfer to the patient. A human-derived feeder fibroblast cell line could be banked and screened for adventitious agents. However, in addition to removing any risk of disease transfer, using autologous feeder fibroblasts for autologous epithelial expansion would also remove the need for immunosuppression. The aim of this study was to investigate whether human derived limbal or oral mucosal fibroblasts could be used as alternative feeders to the gold standard 3T3 for the expansion of epithelial cells for use in the clinic. HLF and HOMF were studied for their ability to support the expansion of HLE and HOME. Autologous HLF could be obtained for patients with unilateral LSCD, and autologous HOMF for patients with unilateral or bilateral LSCD. To our knowledge the use of HOMF as a feeder fibroblast for the culture of epithelial cells for the treatment of LSCD has not previously been reported, nor has using HLF as a feeder fibroblast for HOME. The use of HLF as a feeder fibroblast for HOME was investigated to see if these cells would be useful for inducing a more corneal phenotype in HOME. 


\section{Methods}

HLE and HOME were initially expanded in T25 tissue culture flasks on Mitomycin C growth arrested 3T3 (primary culture) and cryopreserved. Following cryopreservation, the epithelial cells were split equally (1:3) between the following: 1) T25 with growth arrested HLF, 2) T25 with growth arrested HOMF, and 3) T25 with growth arrested 3T3. Epithelial cells were then passaged (at a seeding density of $1.5 \times 10^{4}$ epithelial cells $/ \mathrm{cm}^{2}$ ) onto the same fibroblast feeder type until they ceased to proliferate. Colony forming efficiency assays were set up at each passage. Protein and RNA were isolated from epithelial cells for PCR and Western blotting experiments. RNA was isolated from passage 2 epithelial cells. Protein was isolated from passage $1 \mathrm{HLE}$ (cultured as described), and from passage $3 \mathrm{HOME}$ with the following alteration to the culture method: HOME cultured on 3T3 for primary and passage 1 expansions prior to being split equally between 3T3, HLF and HOMF feeder layers (passage 2).

Initial expansion on 3T3 was performed to enable scheduling of all the different feeder layers to be ready at the same time for primary epithelial cells to be passaged onto as supply of limbal tissue is not predictable, nor is the exact length of primary culture time to reach confluency. Also, we had a limited supply of HOMF if low/comparable passage numbers were to be used for the duration of the experiment. HLE were also co-cultured with HOMF from isolation (primary culture) to see if epithelial cultures could be initiated on HOMF.

\section{Isolation and culture of HLE:}

HLE were isolated from cadaveric limbal rims (in accordance with EU research consent and ethics approval requirements). Each rim was immersed in $1.2 \mathrm{U} / \mathrm{ml}$ Dispase II (Roche Diagnostics) for either 1-2 hours at $37^{\circ} \mathrm{C}$ or overnight at $4{ }^{\circ} \mathrm{C}$. The rim was removed from Dispase and the epithelial cells scraped from the limbal region of the tissue. Cells were then suspended in Corneal Epithelial Culture Medium (CECM) and seeded into a T25 tissue culture flask containing growth arrested 3T3 (for Figure 7, HLE were seeded onto growth arrested HOMF). CECM consists of the following: 3:1 Dulbecco's Modified Eagle Medium: Nutrient Mixture F-12 (DMEM:F12) (Life Technologies) with 10\% fetal bovine serum (FBS) (Life Technologies), $0.4 \mu \mathrm{g} / \mathrm{ml}$ hydrocortisone (Sigma), $0.1 \mathrm{nmol}$ cholera toxin (Sigma), $5 \mu \mathrm{g} / \mathrm{ml}$ insulin (Sigma), $1 \mathrm{x}$ antibiotic/antimycotic (Life Technologies), $0.18 \mathrm{mmol}$ adenine (Sigma), $2 \mathrm{nmol}$ triiodothyronine (T3) (Sigma), $5 \mu \mathrm{g} / \mathrm{ml}$ transferrin (Sigma), and 10ng/ml epidermal growth factor (EGF) (Life Technologies) plus $0.075 \%$ sodium bicarbonate (Life Technologies). Culture medium was changed three times a week and HLE were passaged before they became confluent. All cells were cultured in the presence of $5 \% \mathrm{CO}_{2}$ in air at $37^{\circ} \mathrm{C}$.

\section{Isolation and culture of HOME}

Oral mucosal biopsies were taken from healthy volunteers (with appropriate research consent and ethics approval). Patients were asked to rinse their mouth with chlorhexidine $0.2 \%$ mouthwash (Corsodyl, GlaxoSmithKline Ltd) for 10 minutes and biopsies were harvested under local anaesthetic. Briefly $1 \%$ tetracaine was applied topically to the buccal mucosa using cotton buds followed by submucosal injection of $0.3 \mathrm{ml}$ Xylocaine $2 \%$ with adrenaline $(1: 200,000)$ Astra Zeneca UK Ltd. A $3 \mathrm{~mm}$ diameter punch biopsy (Stiefel) was used to trephine through the epithelium into the submucosa. The epithelium and a small amount of superficial submucosal tissue was then excised using a toothed forceps and Westcott scissors (Duckworth and Kent, UK). Each oral mucosal biopsy was immersed 3 times in disinfection solution: D-PBS (Life Technologies) + 0.5X antibiotic - 
antimycotic (diluted from 100X antibiotic-antimycotic containing 10,000units/ml Penicillin, $10,000 \mu \mathrm{g} / \mathrm{ml}$ Streptomycin and $25 \mu \mathrm{g} / \mathrm{ml}$ Amphotericin B, Life Technologies) plus $5 \mu \mathrm{g} / \mathrm{ml}$ Amphotericin B (Sigma) for 10 mins at room temperature. Biopsies were subsequently placed in 1.2IU Dispase II (Roche Applied Science) and incubated at $37^{\circ} \mathrm{C}$ for 1 hour. The biopsy was then treated with $0.25 \%$ trypsin:EDTA (diluted from $0.5 \%$ trypsin:EDTA from Life Technologies) for 30mins at room temp. Culture medium (CECM) was used to quench the trypsin and the tissue gently scraped using forceps. The resulting cell suspension was centrifuged for 5 mins and the resulting pellet resuspended in culture medium and plated onto a Mitomycin C growth arrested 3 T3 feeder layer in a T25 tissue culture flask. Culture media was changed three times a week and HOME passaged before they became confluent.

HOME were also cultured in serum-free media for 2 reasons. Firstly to confirm that PAX6 expression was not due to any fibroblasts remaining in the HOME samples tested, and secondly to see if PAX6 expression was a result of using fibroblasts in the culture process. PAX6 expression was compared for HOME obtained using the epithelial-fibroblast co-culture method, the feeder-free culture method, and also for fibroblast only samples (HLF, HOMF, and 3T3). For keratinocyte serum free medium (KSFM) cultured HOME, the following procedure was used: HOME were isolated using the same procedure described above but HLF medium was used to quench the trypsin, cells were resuspended in defined KSFM (Life Technologies) following centrifugation and plated onto a T25 flask coated with collagen type I (rat tail) (First Link UK Ltd). Flasks were coated with $0.3 \mathrm{mg} / \mathrm{ml}$ collagen in $3 \%$ acetic acid for 1 hour at $37^{\circ} \mathrm{C}$, and washed three times with PBS prior to seeding HOME on top. Upon reaching $70 \%$ confluence, cells were detached using $0.05 \%$ trypsin:EDTA at $37^{\circ} \mathrm{C}$ for 10 minutes, quenched with HLF medium, spun down and re-suspended in KSFM before being split 1:3 onto T25s coated with collagen I. Once these passage 1 cultures were $70 \%$ confluent, protein and RNA were extracted for PCR and Western blotting experiments.

\section{Differential trypsinisation for removal of fibroblasts from epithelial cultures:}

Epithelial cells and fibroblasts were separated from cultures using differential trypsinisation in which 1xtrypsin:EDTA is firstly used to remove the fibroblasts. The epithelial cells remain attached to the tissue culture surface and are subsequently detached using 10x trypsin:EDTA [12]. Cultures are thoroughly observed under a microscope to ensure that only cells with an epithelial phenotype remain attached to the tissue culture plastic following the first trypsinisation step.

When epithelial cells (HLE and HOME) were ready for passaging, differential trypsinisation was used to remove feeders (3T3, HLF and HOMF) from the culture and obtain an epithelial cell suspension. CECM was added to the harvested epithelial cells prior to centrifuging at 80-100g for 5 minutes. The cell pellet was then re-suspended in CECM and the epithelial cells ready to be seeded onto a growth arrested feeder layer.

\section{Isolation and culture of HOMF}

Following isolation of HOME from buccal oral mucosal biopsies, the remaining tissue was explant cultured in the bottom of a 6 well plate to produce HOMF using the following culture media: DMEM + glutamax, 10\% FBS, and 1x antibiotic antimycotic (Life Technologies). Following initial expansion, these fibroblasts were passaged at a ratio of 1:3. 


\section{Isolation and culture of HLF}

Following isolation of HLE from limbal tissue, explant cultures were set up for limbal fibroblasts. The explants from one rim were placed in three T25 tissue culture flasks. Explants were cultured in DMEM+glutamax with $10 \%$ FBS and $1 x$ antibiotic antimycotic. Following initial expansion, these fibroblasts were passaged at a ratio of 1:3.

\section{Culture of $3 T 3$}

3 T3 $\mathrm{J} 2 \mathrm{~s}$ were cultured in DMEM + glutamax, $10 \%$ adult bovine serum (ABS), and $1 \mathrm{x}$ antibiotic antimycotic (Life Technologies) and passaged at a ratio of up to 1:8.

\section{Growth arrest of fibroblasts.}

$3 \mathrm{~T} 3$ fibroblasts were treated with $4 \mu \mathrm{g} / \mathrm{ml}$ Mitomycin C (Movianto or Sigma) in 3T3 culture medium for 2 hours at $37^{\circ} \mathrm{C}$ with $5 \% \mathrm{CO}_{2}$. $\mathrm{HLF}$ and $\mathrm{HOMF}$ were treated with $10 \mu \mathrm{g} / \mathrm{ml}$ Mitomycin C in culture medium for 3 hours at $37^{\circ} \mathrm{C}$ with $5 \% \mathrm{CO}_{2}$ in culture media. Fibroblasts were then washed with Phosphate-Buffered Saline (PBS), and detached from the tissue culture flask using 1xtrypsin:EDTA. Culture medium was then added to the growth arrested fibroblasts which were then centrifuged at 80-100g for 5 minutes, and the supernatant removed. $3 T 3$ were seeded at $2.7 \times 10^{4} \mathrm{cells} / \mathrm{cm}^{2}$, and HLF and HOMF at $1.2 \times 10^{4}$ cells $/ \mathrm{cm}^{2}$. Cells were allowed to attach for at least 2 hours, and the culture media replaced with CECM before seeding epithelial cells on top.

\section{Population doublings}

The number of population doublings was calculated using the following equation which takes into account the colony forming efficiency of the cells [13]:

Number of population doublings $=3.322 \log \left(\mathrm{N} / \mathrm{N}_{0}\right)$.

Where $\mathrm{N}=$ the total number of cells obtained at each passage and $\mathrm{N}_{0}=$ the number of clonogenic cells. Colony forming efficiency assay was performed as follows:

For the CFE assay 100-1000 HLE or HOME were seeded per well of a six well plate containing $2 \times 10^{5}$ growth arrested $3 \mathrm{~T} 3$ per well. Cells were cultured for 7 to 12 days in CECM and supplemented with additional feeders if necessary. Cells were fixed with ice-cold methanol and stained with $2 \%$ rhodamine B (Sigma). The total number of colonies was counted using Image J software, and the following equation was used to calculate the colony forming efficiency:

CFE $(\%)=($ Number of colonies/Number of epithelial cells seeded $) \times 100$

The population doublings during the primary culture were not included in the calculation since epithelial cells were initially expanded on 3T3. The number of population doublings calculated is from passage 1 onwards when the epithelial cells were split equally between the 3 different feeder fibroblast groups. Thus the number of population doublings described in this study refers to those obtained on that particular feeder layer only following primary expansion on 3T3 to ensure results are comparable. Population doublings were calculated for multiple passages of epithelial cells from passage 1 to senescence. 
Polymerase chain reaction (PCR)

\begin{tabular}{|c|c|c|c|c|c|}
\hline Primer & Sequence & $\begin{array}{l}\text { Annealing } \\
\text { temp }\left({ }^{\circ} \mathrm{C}\right)\end{array}$ & $\begin{array}{l}\text { Number } \\
\text { of } \\
\text { cycles }\end{array}$ & $\begin{array}{l}\text { Product } \\
\text { size }\end{array}$ & $\begin{array}{l}\text { Sequence } \\
\text { reference }\end{array}$ \\
\hline ABCG2 & $\begin{array}{l}\text { F: AGTTCCATGGCACTGGCCATA } \\
\text { R: TCAGGTAGGCAATTGTGAGG }\end{array}$ & 46 & 32 & 379 & [14] \\
\hline CK12 & $\begin{array}{l}\text { F: } \\
\text { ACATGAAGAAGAACCACGAGGATG } \\
\text { R: TCTGCTCAGCGATGGTTTCA }\end{array}$ & 60 & 35 & 150 & [15] \\
\hline CK15 & $\begin{array}{l}\text { F:GGAGGTGGAAGCCGAAGTAT } \\
\text { R: GAGAGGAGACCACCATCGCC }\end{array}$ & 60 & 32 & 194 & {$[16]$} \\
\hline$\Delta \mathrm{Np63 \alpha}$ & $\begin{array}{l}\text { F: GGAAAACAATGCCCAGACTC } \\
\text { R: ATGATGAACAGCCCAACCTC }\end{array}$ & 60 & 35 & 1389 & [17] \\
\hline GAPDH & $\begin{array}{l}\text { F: GCCAAGGTCATCCATGACAAC } \\
\text { R: GTCCACCACCCTGTTGCTGTA }\end{array}$ & 55 & 35 & 498 & [15] \\
\hline MUC16 & $\begin{array}{l}\text { F: } \\
\text { GCCTCTACCTTAACGGTTACAATGAA } \\
\text { R: GGTACCCCATGGCTGTTGTG }\end{array}$ & 60 & $35 / 30$ & 114 & [18] \\
\hline PAX6 & $\begin{array}{l}\text { F: ATA ACC TGC CTA TGC AAC CC } \\
\text { R: GGAACTTGAACTGGAACTGAC }\end{array}$ & 55 & 35 & 208 & [15] \\
\hline
\end{tabular}

Table 1. Primers used for PCR.

Fibroblasts were removed from cultures by differential trypsinisation with 1Xtrypsin:EDTA prior to harvest of the epithelial cells with 10Xtrypsin:EDTA. RNA was isolated from cells using the RNeasy Plus Mini Kit (Qiagen GmbH, Hilden,Germany) and quantified using an Eppendorf Biophotometer. Of each sample, $1 \mu \mathrm{g}$ was used to synthesise cDNA using the Roche AMV reverse transcriptase kit (Roche Diagnostics GmbH, Mannheim, Germany) following treatment with DNase I (Life Technologies) and subsequent inactivation of the enzyme. Alternatively the Quantitect Reverse Transcriptase kit (Qiagen) was used (figure 6). Equal amounts of CDNA were used for PCRs using the primers listed in Table 1. Each cDNA product (1 $1 \mu \mathrm{l})$ was mixed with the following: $1 \mu \mathrm{l} 5 \mu \mathrm{M}$ forward primer, $1 \mu \mathrm{l} 5 \mu \mathrm{M}$ reverse primer, and $6 \mu \mathrm{l}$ MegaMixBlue (Helena Biosciences). The thermocycling program used was: $94^{\circ} \mathrm{C}$ for 2 minutes, then 35 cycles (or other as indicated in table 1 ) of the following: 30 seconds at $94 \circ \mathrm{C}, 30$ seconds at the annealing temperature, and 1 minute at $72 \circ \mathrm{C}$. The final step was 7 minutes at $72^{\circ} \mathrm{C}$. Agarose gel electrophoresis was used to visualise PCR products, with Hyperladder II (Bioline Reagents Ltd) as a molecular weight marker. No sample and no reverse transcriptase controls were also performed.

\section{Western blotting}

Fibroblasts were removed from cultures using 1xtrypsin:EDTA prior to protein extraction from epithelial cells. Protein was extracted using RIPA buffer (Thermo Scientific) plus Halt Protease Inhibitor and EDTA (Thermo Scientific), and quantified using the Pierce BCA protein assay kit (Thermo Scientific). Western blotting with $40 \mu \mathrm{g}$ of each sample ( $25 \mu \mathrm{g}$ for figure 6 ) was performed as previously described [19] but with $3 \times 10$ minute washes rather than $3 \times 20$ minute washes for the following antibodies: Cytokeratin 15 (CK15) (Abcam, AB52816), PAX6 (rabbit, Covance, PRB-278P), and GAPDH (Millipore, MAB374). For Mucin 16 (MUC16) (Abcam, AB134093) the following buffer was used for blocking and primary antibody incubation: TBST (1xTBS, $0.1 \%$ Tween 20$)+5 \%$ BSA. TBST was used as a dilution buffer for the secondary antibody incubation step. For p63 $\alpha$ (Cell Signaling Technology, 4892) manufacturer's instructions were followed for blocking, washing and antibody incubation steps. Primary antibody dilutions and the predicted molecular weights of the protein 
bands of interest are shown in table 2 . The secondary antibodies used were goat anti-mouse HRP (P0447, Dako) (1:10,000) and goat anti-rabbit HRP (P0448, Dako) (1:5000-1:10,000). Image J software was used for densitometric analysis, and results normalised to GAPDH. Results were obtained from 3 different donors ( 3 HLE donors, and 3 HOME donors) for each epithelial-fibroblast combination. For each gel, one set of HLE protein samples (i.e. from the same donor) cultured with the 3 different feeder types was loaded alongside one set of HOME protein samples to allow comparison. Results from the $3 \mathrm{HLE}$ and $3 \mathrm{HOME}$ experiments were normalised to the gold standard HLE-3T3 culture and one way ANOVA performed.

\begin{tabular}{|l|l|l|l|}
\hline Antibody & Raised in & Dilution & Predicted Mwt in kDa \\
\hline CK15 & Rabbit & $1: 10,000$ & 45 (observed 45 and 50 bands) \\
\hline GAPDH & Mouse & $1: 1500$ & 38 \\
\hline MUC16 & Rabbit & $1: 10,000$ & 2353 (observed bands starting at approximately 140) \\
\hline p63 $\alpha$ & Rabbit & $1: 800-1: 1000$ & 75 \\
\hline PAX6 & Rabbit & $1: 500$ & $47-50$ \\
\hline
\end{tabular}

Table 2. Antibody dilutions used for western blotting and the predicted molecular weights of the protein bands of interest from the manufacturers datasheets. Where bands observed in this study differed from the predicted size this is indicated.

\section{$\underline{\text { Results }}$}

\section{Passage number and Population doublings}

All epithelial types were successfully expanded on all fibroblast feeder layers as shown in Figure 1. Oral fibroblasts were comparable to 3T3 as a feeder layer for both limbal and oral epithelial cells in terms of the number of population doublings (Figure 2) with no significant difference between these feeder types. The number of epithelial population doublings (both oral and limbal) achieved with limbal fibroblasts as a feeder layer was significantly lower $(P<0.05)$ than with the gold standard 3T3.

The average (mean) number of passages obtained for limbal epithelial cells cultured on 3T3, limbal and oral fibroblasts was 7, 3 and 6.7 respectively. Higher passage numbers were obtained for oral epithelial cells cultured on the 3 different feeder layers with the average (mean) passage numbers being 10, 5 and 10.3 for 3T3, limbal and oral fibroblasts respectively.

For HLE, the mean number of population doublings obtained with 3T3, HLF and HOMF was 38.4, 9.3, and 35.1 respectively. For HOME these values were $41.7,19.3$, and 52.1 respectively.

\section{Stem cell marker expression}

PCR showed that the putative stem cell markers p63 $\alpha$, ABCG2, and CK15 were present in all cellular combinations studied (Figure 4) Western blot showed protein expression of p63 $\alpha$ and CK15 in all conditions (Figure 5). For CK15, two bands of approximately 50kDa and 45kDa in size were observed by western blot. The upper band $(50 \mathrm{kDa})$ was observed in both oral and limbal epithelial cells, but the lower band $(45 \mathrm{kDa})$ was present only in the limbal cultures. A431 (human epidermoid carcinoma cell line) cell lysate (Santa Cruz Biotechnology) was used as a positive control for CK15 and also had two bands. Protein expression of $\mathrm{p} 63 \alpha$ was not significantly different for any of the epithelialfibroblast combinations (one way ANOVA). CK15 was significantly lower in oral epithelial cells expanded on all 3 feeder fibroblast types compared to limbal epithelial cells co-cultured with 3T3s 
$(P<0.05)$, but was not significantly different for limbal epithelial cells cultured on the different feeder fibroblasts. Oral epithelial cells co-cultured with oral fibroblasts had significantly lower CK15 compared to when cultured on 3T3s $(P=0.0299)$.

\section{Corneal marker expression}

PAX6 and CK12 are expressed in the cornea and therefore their gene expression was determined to see if limbal fibroblasts could drive the oral epithelial cells towards a more corneal phenotype in culture. PAX6 mRNA was expressed in oral cells irrespective of the fibroblast feeder layer albeit at a "low signal level"; western blotting also showed a faint band for PAX6 in these cultures (Figure 4). CK12 mRNA was however not observed in oral cells under any conditions (Figure 4). Since several studies have used oral epithelium as a negative control for PAX6 [15, 20], these cells were cultured in feeder-free, serum-free media (defined KSFM) and protein analysed to see if the low PAX6 expression was a result of using fibroblasts as a feeder layer. However, PAX6 protein was still detected in these KSFM cultured oral epithelial cells; in addition, no protein was detected in any fibroblast type (Figure 6).

MUC16 has been shown to be expressed in tissue sections of the corneal epithelium, but not in the oral mucosal epithelium [21]. In this study we observed high MUC16 protein expression in limbal epithelial cells co-cultured with all fibroblast types (Figure 5). MUC16 protein expression in oral epithelial cells was also observed on all fibroblast feeder layers (Figure 5) however it was highly variable with cells cultured on 3T3 demonstrating expression in cultures derived from only one of the donors and low levels expressed in cells from all donors cultured on limbal or oral fibroblasts. These levels of MUC16 protein expression were all significantly lower than those observed in limbal epithelial cells cultured on limbal fibroblasts $(P<0.05$, One way ANOVA, Bonferroni's multiple comparison test). MUC16 mRNA was expressed in all epithelial-fibroblast co-cultures studied (Figure 4).

\section{Epithelial cultures initiated using HOMF as a feeder layer}

HOMF were observed to support the primary expansion of HLE as shown in Figure 7. HLE cultures were set up from 5 eyes from 4 different donors. 4 of the 5 cultures reached confluency by day 6 . The culture that did not reach confluency by this time was initiated from one of a pair of donor eyes of which HLE from the other one reached confluency by day 6 perhaps suggesting poor tissue quality since all other culture conditions were the same including HOMF. Thus, we observed successful primary epithelial expansion on HOMF for 4/4 different HLE donors. 

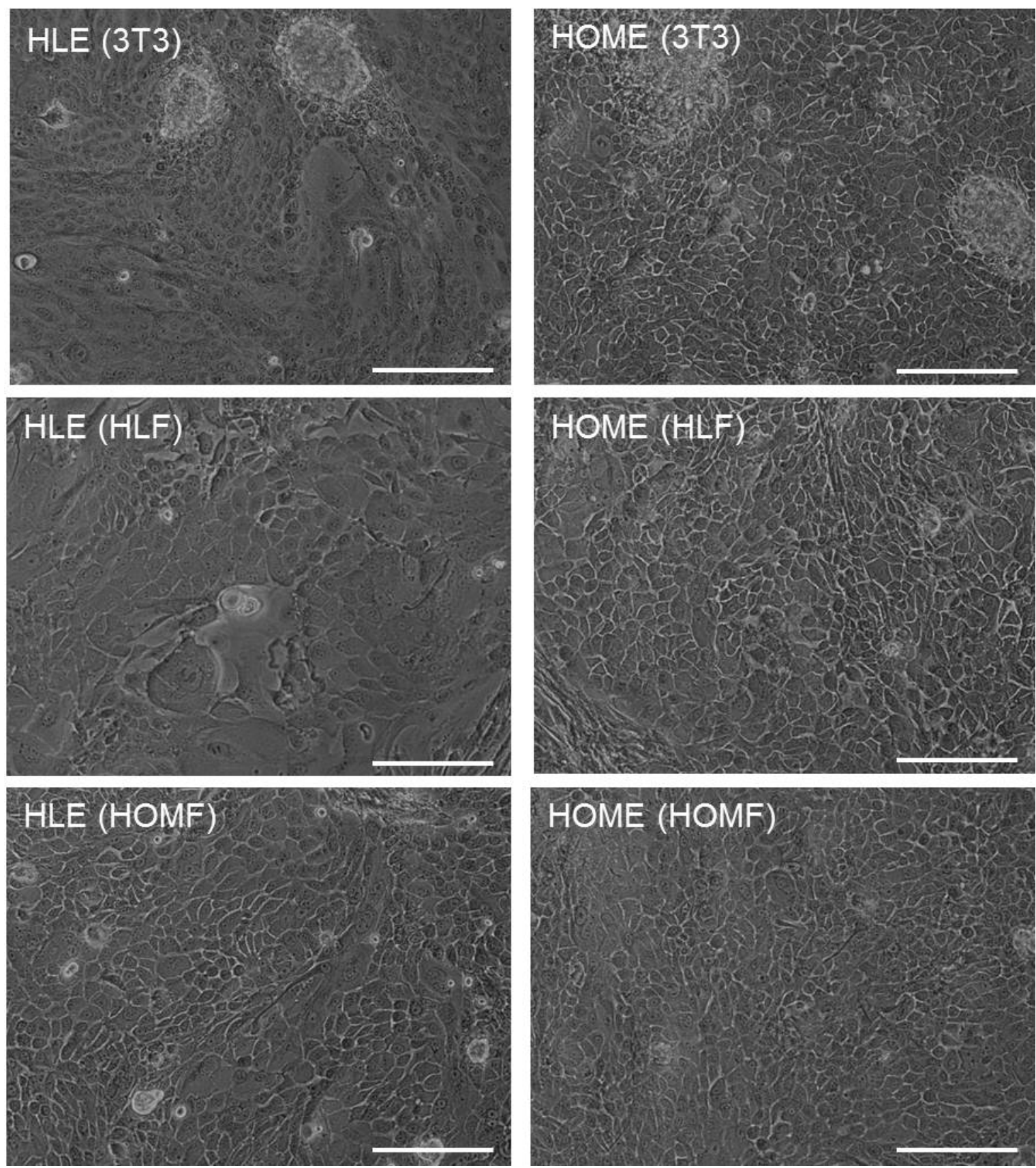

Figure 1. HLE and HOME can be successfully expanded using 3T3, HLF, or HOMF as feeder

fibroblasts. Cells with epithelial morphology were observed for all cultures with areas of epithelial cells shown here for each epithelial-fibroblast co-culture. Images for HLE are of cells from the same HLE donor. Images for HOME are of cells from the same HOME donor. Images were taken at passage 1. Scale bar $=200 \mathrm{micron}$. 

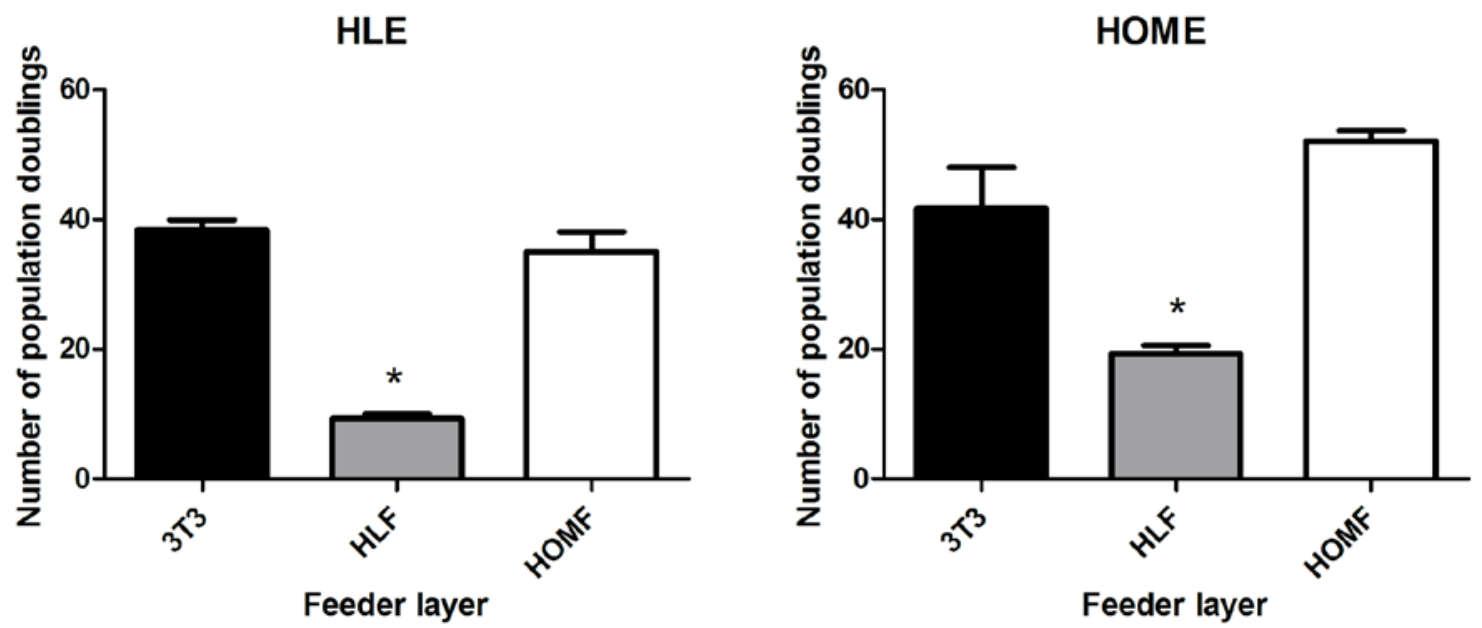

Figure 2: Population doublings obtained for HLE and HOME expanded on 3T3, HLF or HOMF feeder layers. Epithelial cells were initially expanded on 3T3, and then split equally onto $3 T 3, H L F$, and HOMF feeder layers and cultured on these feeder layers until they ceased to proliferate any further. Population doublings were calculated from $\mathrm{p} 1$ until senescence i.e. expansion in the primary culture not included. $n=3$ ( 3 HLE donors, and 3 HOME donors). $\mathrm{P}<0.05$, one way ANOVA compared to 3T3 controls. The number of epithelial population doublings observed using HOMF as feeder fibroblasts was comparable to that obtained using the gold standard 3T3 feeder fibroblasts. The number of epithelial population doublings obtained on HLF feeder layers was however significantly lower than that obtained with the gold standard 3T3 expansion method. 
A.
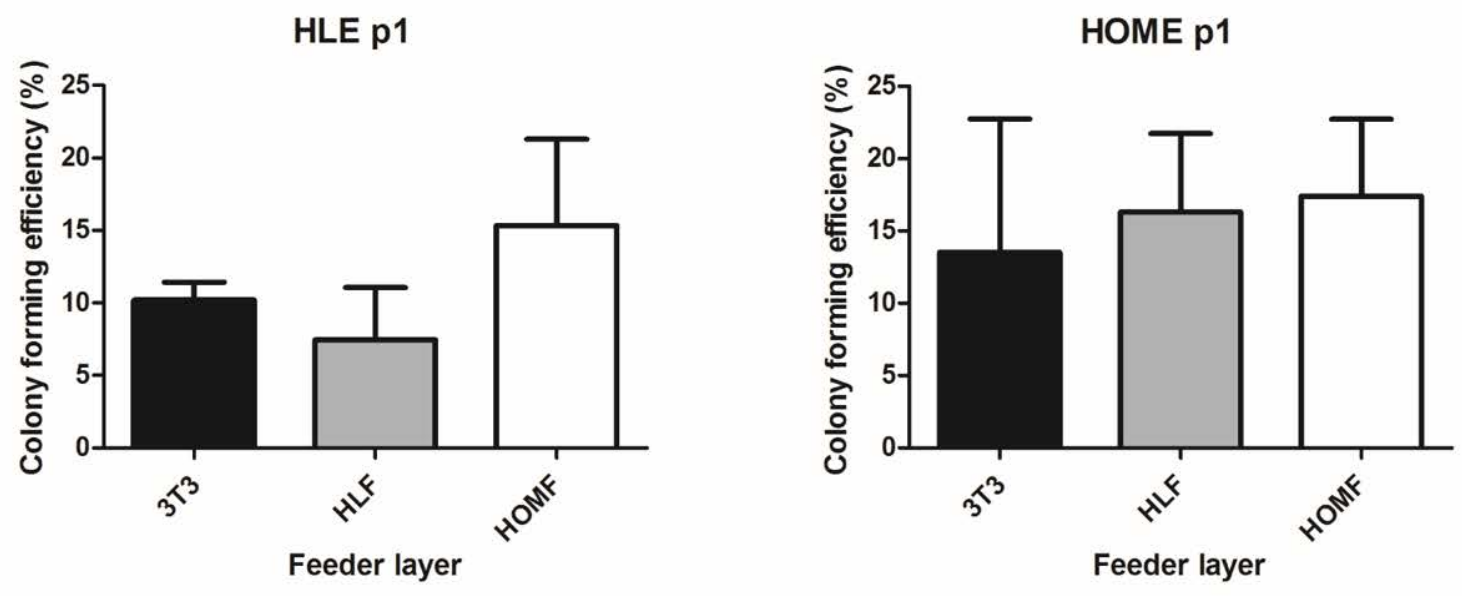

HLE p2
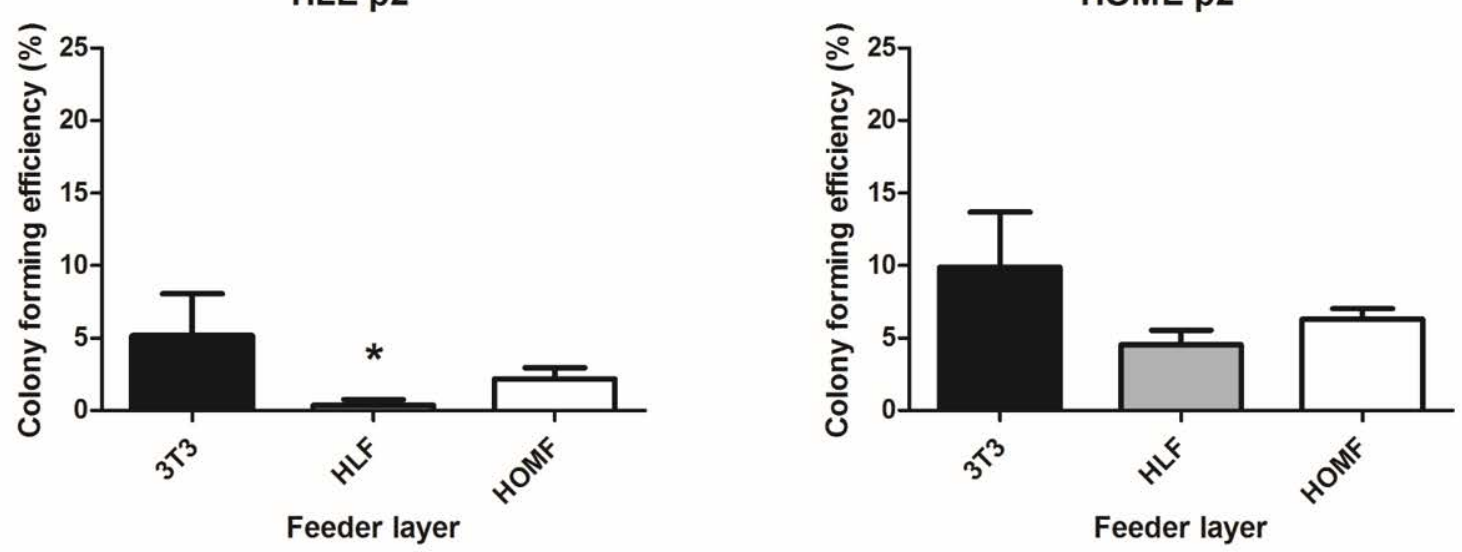

B.
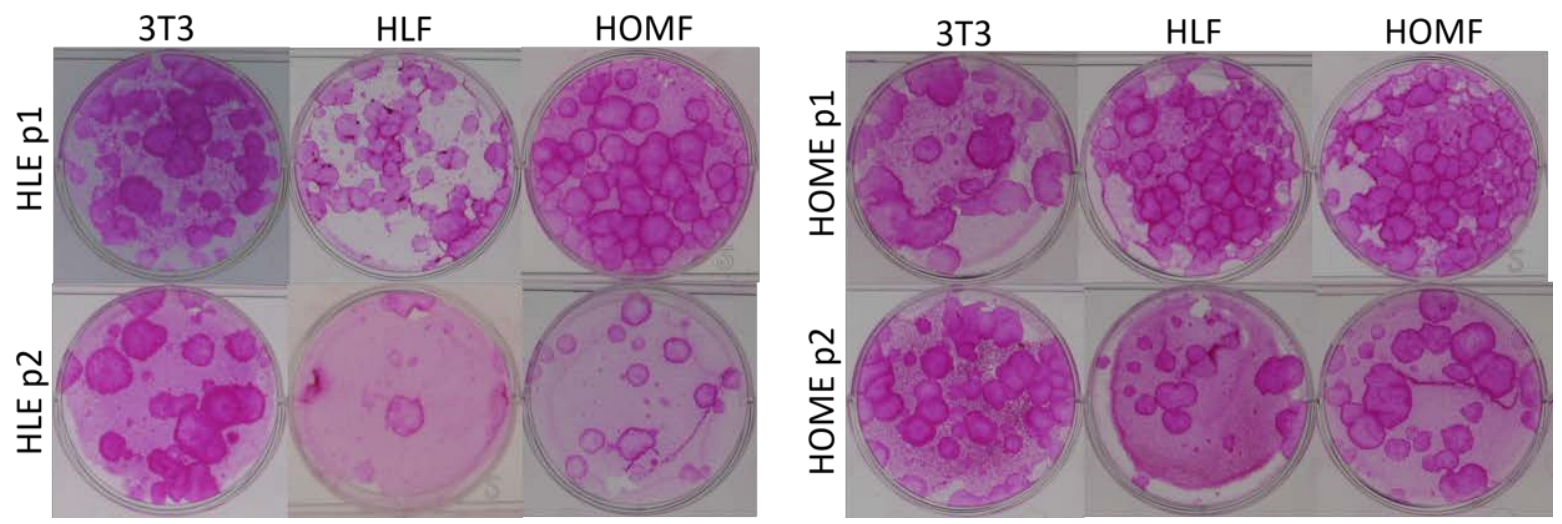

Figure 3. Colony forming efficiency of HLE and HOME cultured on 3T3, HLF and HOMF from

passage 1 and passage 2 cells. A. The number of colonies greater than $2 \mathrm{~mm}$ in diameter was used in the colony forming efficiency calculation thus excluding aborted colonies (paraclones) from the analysis and giving a more accurate measure of proliferative ability than total CFE. Results shown are based on $n=3$ HLE donors, and $n=3$ HOME donors with 3 wells analysed per condition. For HLE, HOMF was comparable to 3T3 for both passage 1 and 2. HLF co-cultured HLE had a significantly lower CFE ( $P<0.05$, one way ANOVA) than $3 T 3$ controls at passage 2. For HOME, no significant 
difference was observed between HOMF or HLF and 3T3. Mean values with SD error bars are shown. B. Examples of colonies obtained from each epithelial-fibroblast group. Results shown are from wells in which 500 epithelial cells were seeded, apart from the passage $1 \mathrm{HOME}$-fibroblast groups where examples shown are with 250 epithelial cells seeded/well.

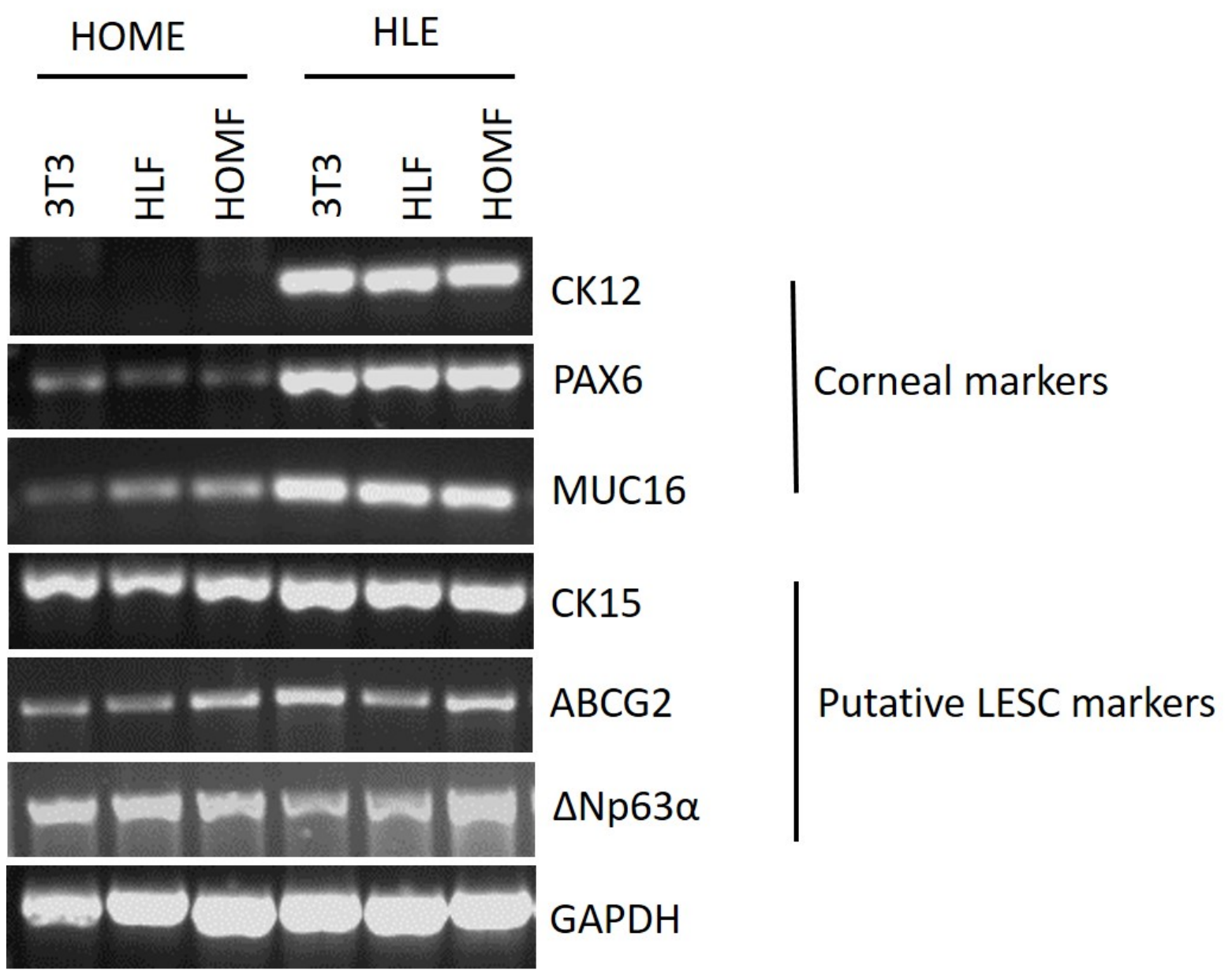

Figure 4. PCR expression of corneal markers and putative stem cell markers for HLE and HOME cultured on 3T3, HOMF, and HLF. The corneal markers PAX6 and MUC16 were detected in epithelial cells (both HLE and HOME) cultured on all three feeder fibroblast types, however CK12 was negative for oral epithelial cells. The putative stem cell markers CK15, ABCG2, and deltaNp63 $\alpha$ were detected in both limbal and oral epithelial cells cultured on all fibroblast types. Representative results are shown. Three experiments were performed with different donors with similar results. 
A.

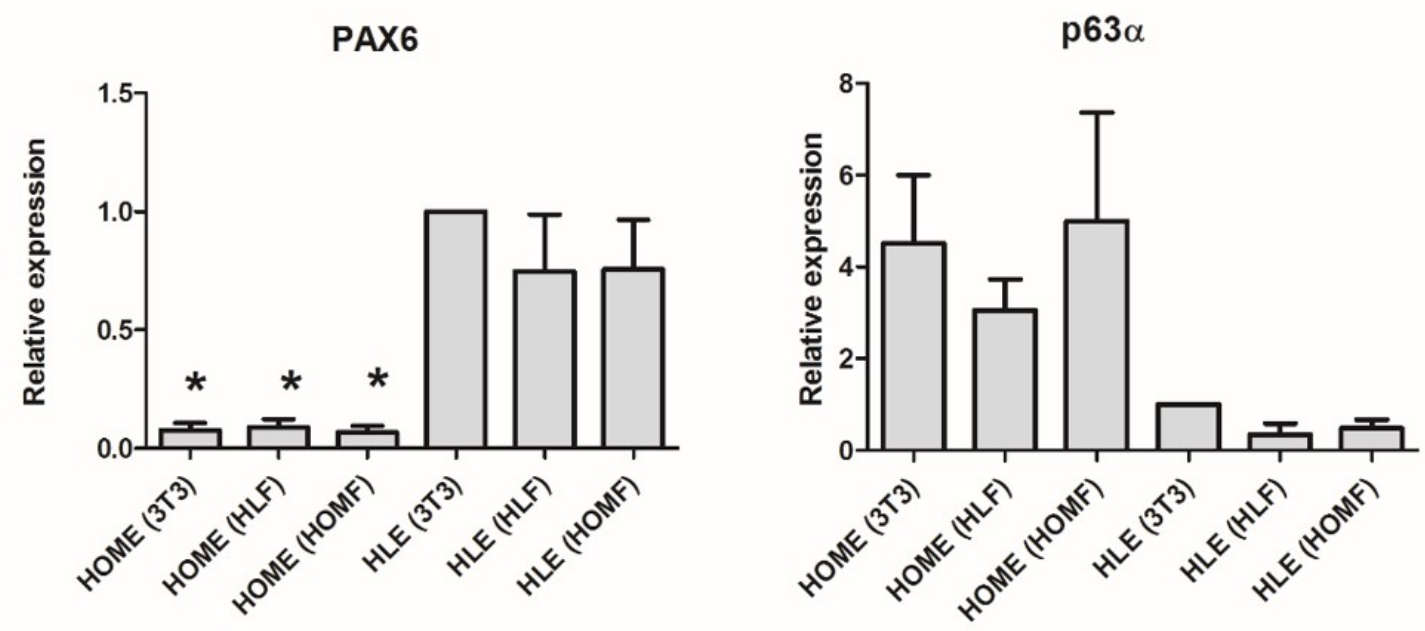

MUC16
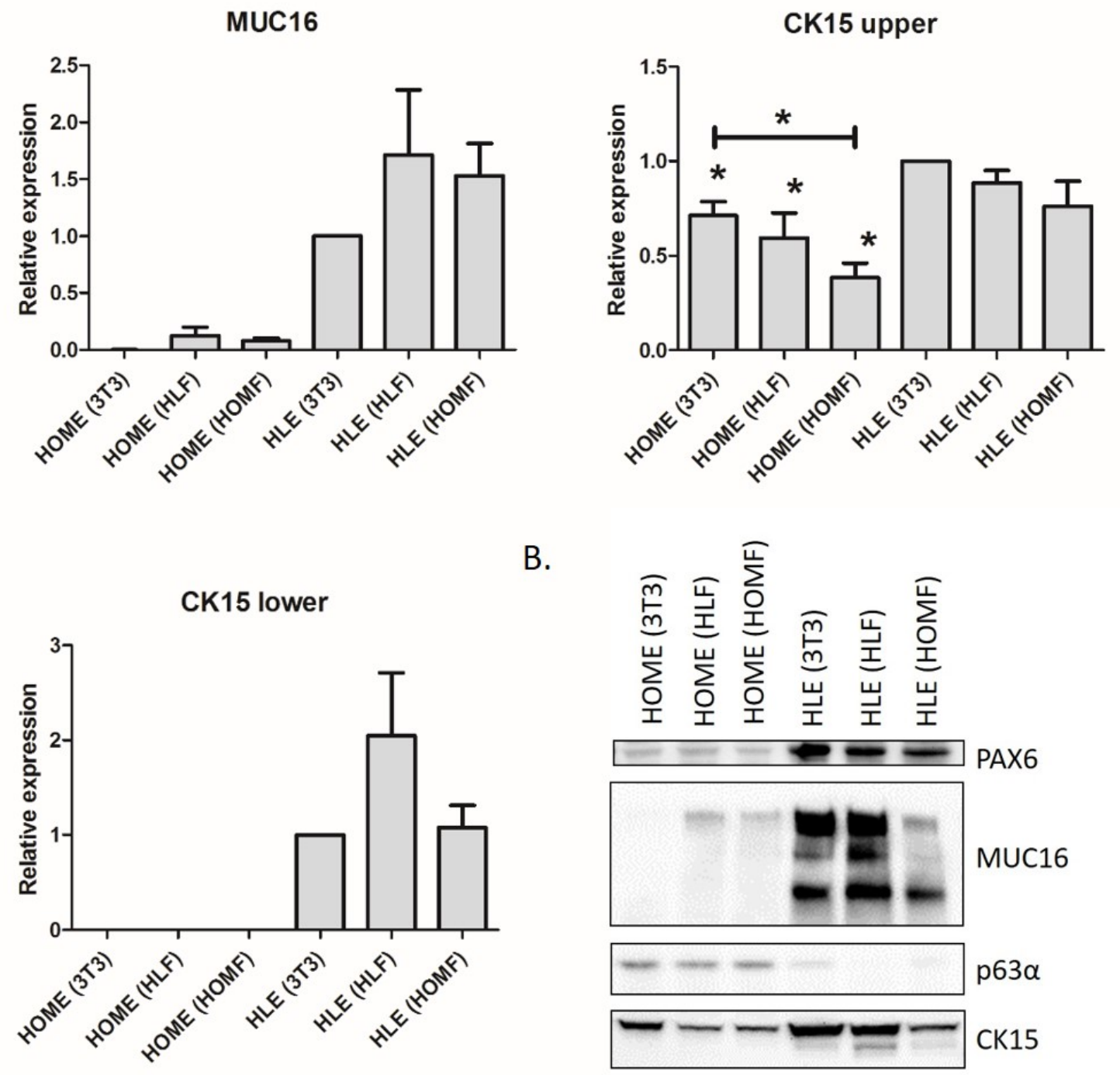

B.

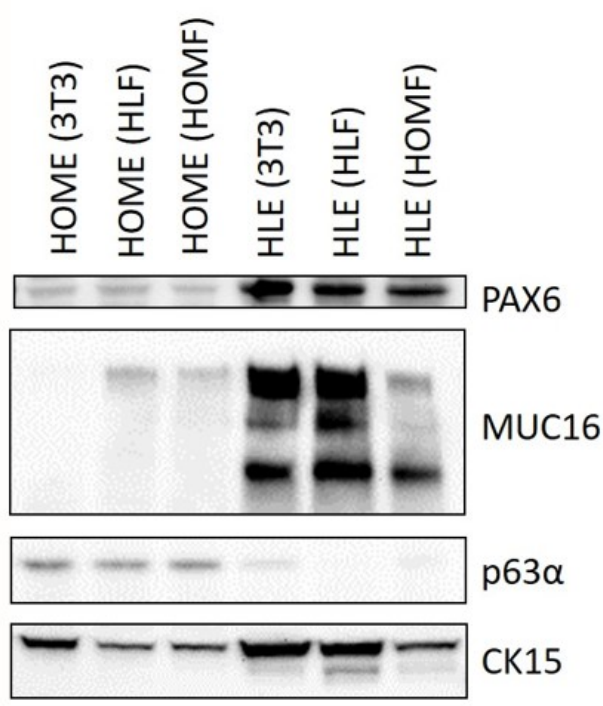

GAPDH 
Figure 5. Western blot analysis of HLE and HOME cultured on different feeder layers: 3T3, HLF, and HOMF. A. Expression of PAX6, p63 $\alpha$, MUC16 and CK15 are shown. These values are normalised to GAPDH and relative to HLE cultured on 3T3. Low levels of PAX6 were observed for HOME cultured on all three feeder fibroblast types, and epithelial PAX6 expression for HLE on HLF or HOMF was comparable to that of HLE on 3T3. P63 $\alpha$ was expressed in higher levels in HOME than HLE. High levels of MUC16 were expressed in HLE cultured on all three feeder fibroblast types. Lower levels of MUC16 were observed for all HOME cultured on HLF or HOMF, and only $1 / 3$ cultures on 3 T3 expressed this protein. Two bands were observed for CK15, a band at approximately 50kDa (CK15 upper) and a band at approximately 45kDa (CK15 lower). The upper band was observed in both limbal and oral epithelial cells whereas the lower band was present only in HLE. For HLE cultured on the 3 different feeder fibroblast types, no significant difference in protein expression was observed between these groups for any of the proteins tested. For HOME cultured on the 3 different feeder fibroblast types, the only significant difference in protein expression observed was between HOME cultured on 3 T3 and HOME cultured on HOMF for CK15 (upper band). ${ }^{*} \mathrm{P}<0.05$ (one way ANOVA) compared to HLE (cultured on a 3 T3 feeder layer) unless otherwise indicated. $\mathrm{N}=3$, apart from the PAX6 graph where $n=4$ HLE donors. B. Example blots are shown for each of the markers tested. 


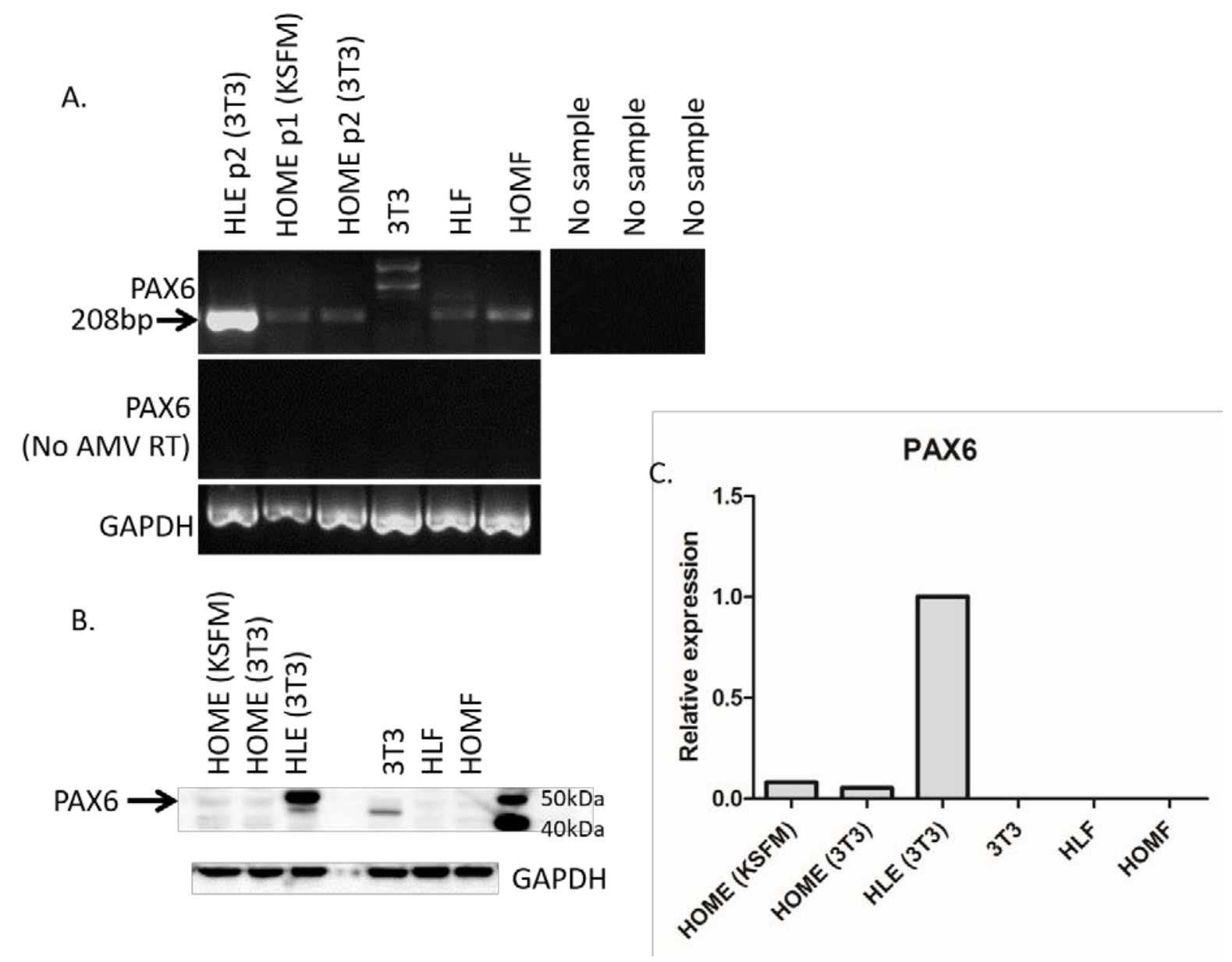

Figure 6. PAX6 expression in HOME cultured with and without feeder fibroblasts. A. PCR expression of PAX6 for the following: HLE and HOME cultured on a 3 T3 feeder layer, HOME cultured in a serumfree feeder-free culture system with KSFM, 3T3, HLF and HOMF. No sample and no AMV reverse transcriptase (no AMV RT) controls were included. Low levels of PAX6 were detected in HOME cultured with and without 3T3, and also in HLF and HOMF. No PAX6 expression was observed in 3T3. B. Western blot showing the expression of PAX6 protein in the various cell types tested. C. Western blot densitometric analysis. PAX6 was normalised to GAPDH and values are relative to HLE-3T3. $\mathrm{N}=1$. Western blotting confirmed that PAX6 was greatly expressed in HLE cells, with low expression in HOME cells cultured with and without 3T3s. No PAX6 protein was detected in any of the feeder fibroblasts tested. 

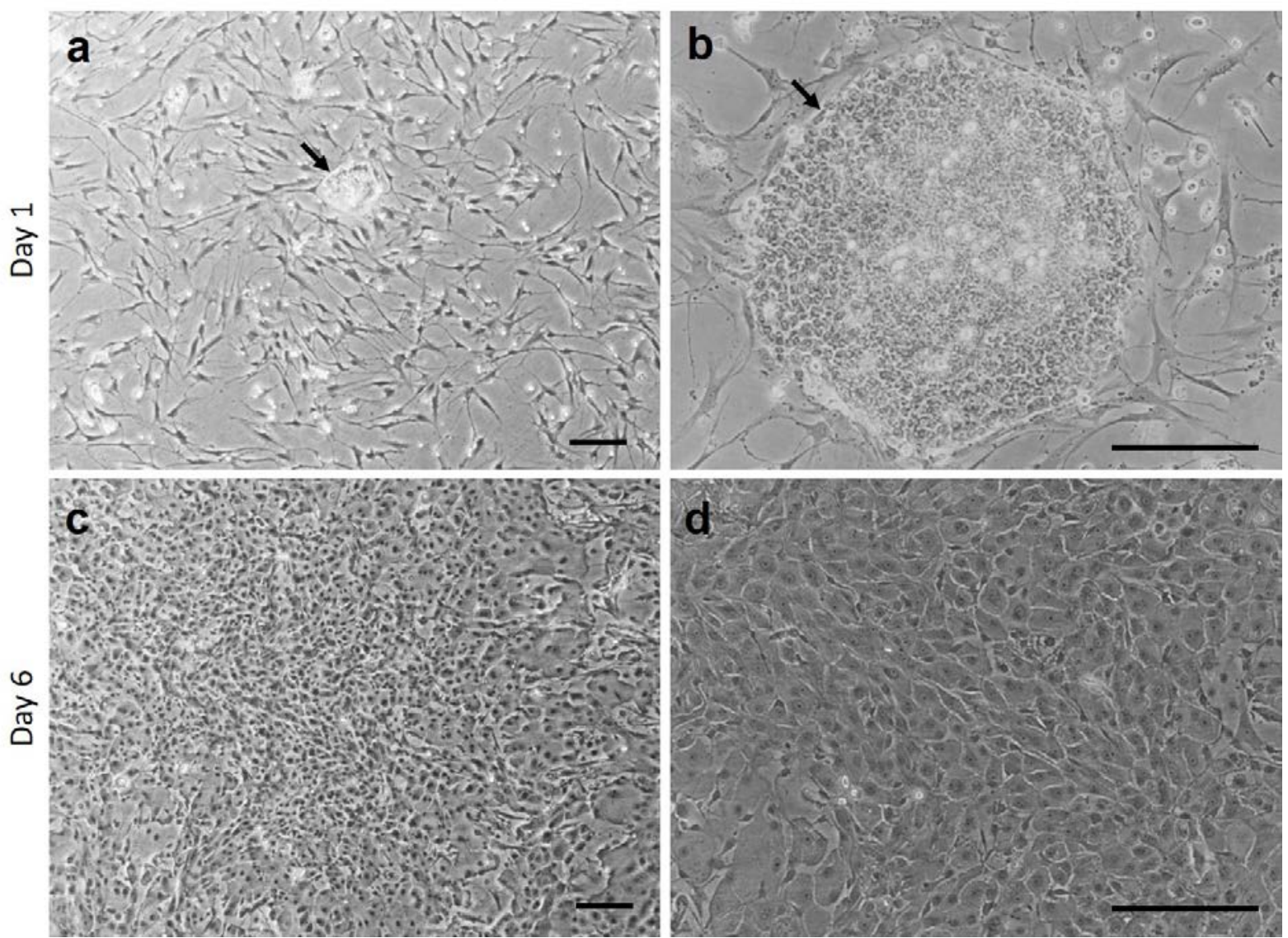

Figure 7. Primary expansion of HLE on HOMF. HOMF were shown to support primary epithelial expansion for 4 different HLE donors. Representative images are shown at day 1 and day 6 . Colonies of HLE consisting of small tightly packed cells were observed on day $1(a, b)$, arrows indicate the epithelial colony edge. Confluent cultures were obtained by day 6 with cells displaying typical epithelial morphology (c, d). Scale bars are $200 \mu \mathrm{m}$.

\section{Discussion:}

Results from this study suggest that human oral mucosal fibroblasts could be used as an alternative feeder fibroblast to 3T3s for producing cultured epithelial cells (derived both from the limbus and the oral cavity) for use in the clinic to treat patients with LSCD. Oral fibroblasts were found to be comparable to 3T3s for use as a feeder fibroblast for expanding both types of epithelial cells tested in terms of number of population doublings, and maintenance of the putative stem cell marker p63 $\alpha$. For limbal epithelial cells, CK15 expression was also comparable when oral fibroblasts and 3T3s were used as a feeder layer as was expression of the corneal markers PAX6, MUC16 and CK12. For oral epithelial cells, expression of the corneal markers PAX6 and MUC16 was also similar following co-culture with either oral or $3 T 3$ feeder fibroblasts.

For the clinic, to eliminate $3 T 3$ completely from the culture system would be ideal. Now we have demonstrated that following primary epithelial expansion on 3T3, HOMF is comparable to $3 T 3$ as a feeder fibroblast for a number of parameters, and may therefore have application for production of clinical grafts the next step would be to perform GMP validation experiments on cultures initiated on HOMF e.g. to assess the percentage of stem cells and the expression of CK12 in the epithelial 
cultures. The number of stem cells in limbal epithelial cultures for the treatment of LSCD is important for clinical success. A minimum of $3 \%$ stem cells (detected as p63-bright holoclone forming stem cells) in such cultures has been shown to be associated with successful transplants [22]. In this study the epithelial cells were cultured as a monolayer. Airlifting to induce cell differentiation and stratification is commonly performed prior to transplantation. The differentiation marker CK12 would be increased in such conditions. Although we have shown here that HOMF can support primary HLE cultures, further validation would be needed to enable their use as a clinical feeder layer.

We used CK15 and p63 $\alpha$ as putative stem cell markers, and MUC16 and PAX6 as corneal markers. CK3 was not used as a corneal marker since CK3 is expressed by HOME as well as HLE [18]. CK12 was not detected by PCR in HOME on any of the feeder layers. We tried various different CK12 antibodies for western blotting for HLE-fibroblast and HOME-fibroblast cultures with no success. However, CK3/12 is expressed in differentiated cells and may therefore be more likely to be detected if stratification had been induced e.g. by airlifting. p63 $\alpha$ antibody was used in this study, p63 $\alpha$ is a stem cell marker expressed only in the basal corneal epithelium. HLE are not maintained in culture as long as HOME as demonstrated in this study (fibroblast co-culture method). By passage $2 \mathrm{HLF}$ cultured HLE have low CFE which correlates with lower p63 $\alpha$ observed for HLE than HOME.

Interestingly we observed low levels of PAX6 in oral epithelial cells cultured on all fibroblast types. Although several studies have shown cultured HOME to be PAX6 negative [15, 20, 23], a recent study found PAX6 expression in human oral mucosal tissue [24]. More specifically, faint pan-nuclear PAX6 staining was observed in the oral mucosal epithelium [24]. Since PAX6 is expressed in the oral mucosal epithelium in vivo, cultured HOME should have the potential to express PAX6 which was shown in this study. PAX6 is important for maintaining a normal corneal epithelial phenotype and is downregulated or absent in epithelial cells from patients with various ocular surface diseases [20]. It therefore seems advantageous for HOME used for COMET to express PAX6.

Mucins are important for maintaining the tear film on the surface of the eye which provides lubrication and a barrier to pathogen entry. MUC16 is a cell-surface associated mucin expressed in the corneal epithelium and dysregulation of this mucin may be associated with dry eye pathology [25]. Hori et al (2008) have shown that MUC16 is expressed in tissue sections of the corneal epithelium, but not the oral mucosal epithelium. The authors did however state that MUC16 may have been lost during pre-treatment prior to harvest of the oral mucosal tissue. In this study we observed lower MUC16 expression in oral epithelial cells compared to those derived from the limbus; the expression in limbal cells was not significantly different between any of the fibroblast types tested. The maintenance of this mucin in HLE regardless of the feeder fibroblast used for expansion may be important for function of these cells on the eye post-transplantation. MUC16 was also observed by PCR for oral epithelial cells irrespective of which fibroblasts were used as a feeder layer. MUC16 protein was detected in 3/3 HOME samples co-cultured with HLF or HOMF and 1/3 $3 T 3$ co-cultured HOME. Limbal and oral fibroblasts are therefore as good as, and possibly better than $3 T 3$ for use as feeder fibroblasts for supporting oral epithelial cells in terms of MUC16 expression. Other studies have also shown MUC16 expression in cultured HOME $[21,26]$. These studies used 3T3 as a feeder fibroblast, and looked at MUC16 expression in stratified cell sheets. The next iteration of the system described here in which cells were cultured as a monolayer could therefore be the induction of a stratified cell sheet to enhance the expression of MUC16 expression in oral epithelial cells. 
Other studies have shown that human embryonic stem cells and murine hair follicle stem cells can be directed towards a more corneal like lineage using a combination of extracellular matrix and limbal fibroblast conditioned media $[27,28]$. In these studies CK12 [27, 28] and Pax6 [28] were upregulated. Since CK12 was not expressed in any of the oral epithelial cultures in this study, and PAX6 and MUC16 were not significantly different for HOME co-cultured with limbal or epithelial fibroblasts our data suggests that more than just fibroblasts are required to direct HOME towards a more corneal-like phenotype.An advantage of feeder layer pre-expansion of epithelial cells over explant techniques for cell therapy, is the provision of sufficient cells for quality control testing e.g. p63 [22]. Pre-expansion also facilitates greater characterisation of the cells. Epithelial cells for use in the clinic to treat LSCD typically only have one expansion step on 3T3. Although all epithelial cells studied were initially expanded on 3T3 for the primary culture, they were subjected to one or two further expansion steps on the different fibroblast feeder types prior to phenotypic analysis. It is possible that greater differences in epithelial phenotype may be observed if HLE and HOME were expanded directly onto HLF and HOMF feeder layers following isolation. Oral fibroblasts are an attractive source of feeder cells as they can be easily isolated from a biopsy from the readily accessible oral mucosal epithelium, a procedure in which the biopsy area heals quickly with minimal scarring. These oral cells could also be an autologous source of fibroblasts from the patient, which used together with autologous epithelial (limbal or oral) cells would mean the patient would not require immunosuppression to prevent graft rejection. Alternatively, oral fibroblasts cells from one donor (cadaveric or living) could be screened and banked to produce grafts for multiple patients.For the treatment of unilateral LSCD autologous HLE could be expanded on autologous HOMF. For bilateral LSCD treatment autologous HOME could be expanded on autologous HOMF. For both treatment types 2 biopsies would need to be taken from the patient. An oral mucosal biopsy would firstly be required from which to isolate HOMF, and once these are suitably expanded, a second biopsy (either limbal or oral) from which to isolate epithelial cells. In this study HOMF were obtained using explant culture. Using this method of isolation it would take approximately 5 weeks to obtain enough passage $1 \mathrm{HOMF}$ for use as a feeder layer (in this study confluent passage 1 cultures were obtained after 5 weeks in culture, although we used these cells as feeder fibroblasts at a later passage number). Typically 2 weeks are required for epithelial expansion on fibroblasts for CLET or COMET. Thus it may take approximately 7 weeks for graft production. Other methods of fibroblast isolation may be quicker e.g. collagenase digestion, but the population of fibroblasts obtained may be different.

In this study limbal fibroblasts were not as good as the gold standard 3T3 for maintaining HOME and HLE cells in culture. Although protein expression of CK15 and p63 $\alpha$ was not significantly different for HOME or HLE cultured on HLF and 3 T3 at passage 2, HLF were not found to be equivalent to 3 T3 in terms of maintaining the proliferative ability of the epithelial cells. The number of population doublings was significantly less for HLE and HOME cultured on HLF compared to $3 T 3$ and HOMF. It is possible that a different population of fibroblasts from the limbus or cornea may be better as a feeder layer. For example, corneal stromal stem cells have been found to generate a better corneal stromal tissue equivalent than human corneal fibroblasts [29] and may be more successful as a feeder layer than the limbal fibroblasts used here. Since limbal epithelial stem cells are not uniformly distributed along the limbus but preferentially located in certain areas [30-32] fibroblasts from these areas of the limbus may also be better for maintaining epithelial stem cells in culture. 


\section{Conclusion:}

Fibroblasts derived from the human oral mucosa could be used as an alternative feeder fibroblast to murine $3 \mathrm{~T} 3$ for the culture of epithelial cells for use in the clinic; oral fibroblasts can easily be isolated from a small buccal oral mucosal biopsy taken from the patient and this biopsy area heals quickly with minimal scarring. Removal of these animal derived 3T3 from the culture system would improve the safety of the procedure. Another advantage of oral fibroblasts is that they can be autologous. Thus for COMET or CLET, autologous fibroblasts could be used to expand autologous epithelial cells derived either from the eye (limbus) or the mouth (oral mucosa) and thus remove the requirement for immunosuppression and its associated side effects.

\section{Future perspective:}

There are now several human feeder fibroblast types that show promise as being suitable alternatives to $3 \mathrm{~T} 3$ for the expansion of epithelial cells for clinical use. These include: oral mucosal fibroblasts described here, tenon's fibroblasts [7], dermal fibroblasts [8], mesenchymal stem-cell derived feeder cells [9], the embryonic fibroblast cell line MRC-5 [10], and the commercially available newborn foreskin fibroblast cell lines Hs68 and CCD1112Sk [11]. These have yet to be used in production of epithelial cells for the treatment of LSCD but it seems the use of a 3T3 alternative may not be to be too far away. In addition to replacing $3 T 3$ with human feeder fibroblasts for the culture of epithelial cells, animal derived products e.g. bovine serum should also be removed from the culture medium (or human-derived alternatives found) to further improve the safety of cultured cells for patients. The ideal would be to have an autologous cell product produced using a fully defined and animal product free culture system. Investigations are currently underway with the aim of producing cell therapies without the use of animal derived products which are of comparable quality and efficacy to those achieved using the current gold standard approach which utilises 3T3 and FBS.

\section{Executive summary:}

\section{Cultured epithelial cell therapy for the treatment of LSCD}

- $\quad$ LSCD can be successfully treated with cultured epithelial cells derived from the human eye (limbus) or mouth (oral mucosa)

- The current gold standard method for expanding these epithelial cells for clinical use uses a 3 T3 feeder fibroblast layer.

- Ideally the procedure for producing these cells should be animal product free. However 3T3 may be permitted on a case-by case basis until an alternative can be found.

\section{Human oral mucosal fibroblasts as an equivalent, safer, feeder fibroblast to $3 \mathrm{~T} 3$ for epithelial expansion}

- HOMF are human-derived, autologous feeder fibroblasts that can be cultured from an easily accessible oral mucosal biopsy which heals quickly with minimal scarring. 
- Oral fibroblasts are equivalent to 3T3 as a feeder fibroblast for the culture of epithelial cells (derived from both the limbus and the oral mucosa) in terms of total passage number, population doublings, and expression of the putative stem cell marker p63 $\alpha$.

- Expression of the corneal markers PAX6 and MUC16 is maintained when limbal epithelial cells are co-cultured on an oral mucosal fibroblast feeder layer.

- Oral fibroblasts could be used as a feeder layer instead of murine 3T3 for the culture of a variety of epithelial cells for use in cultured stem cell therapy for the treatment of LSCD. This system could improve the safety of these procedures, by utilising an autologous source of feeder cells.

- Oral fibroblasts could potentially be used to culture other epithelial cell types for clinical use.

\section{Financial and competing interests disclosure}

This research was funded by Fight for Sight, the Special Trustees of Moorfields Eye Hospital and the National Institute for Health Research (NIHR) Biomedical Research Centre at Moorfields Eye Hospital NHS Foundation Trust and UCL Institute of Ophthalmology. MPL's contribution to this activity was conducted under the auspices of the National Centre for Sport and Exercise Medicine (NCSEM) England, a collaboration between several universities, NHS trusts and sporting and public bodies. The views expressed are those of the authors and not necessarily those of NCSEM England or the partners involved.

The authors have no other relevant affiliations or financial involvement with any organisation or entity with a financial interest in or financial conflict with the subject matter or materials discussed in this manuscript apart from those disclosed.

No writing assistance was utilised in the production of this manuscript.

\section{Ethical conduct of research}

The authors state that they have obtained appropriate ethical institutional review board approval for this study. Informed written consent was obtained from donors of oral mucosal biopsies. Cadaveric limbal tissue was obtained from Moorfields Lions Eye Bank. This tissue had appropriate research consent given by the donor's next of kin.

\section{Acknowledgements:}

The authors would like to thank Mr Alex Shortt (Moorfields Eye Hospital and University College London) for obtaining the oral mucosal biopsies. 


\section{References:}

References that are of particular significance are indicated: *of interest, and ${ }^{* *}$ of considerable interest.

* Rama et al. Successful transplantation of limbal epithelial cells cultured on fibrin to treat LSCD has been associated with cultures in which more than $3 \%$ of clonogenic cells are p63 bright.

*Scafetta et al. Human Tenon's fibroblasts as a human feeder layer for the culture of limbal epithelial cells.

**Gaddipati. Observed PAX6 expression in oral mucosal epithelial tissue.

**Li et al. Corneal epithelial cells from patients with ocular surface diseases such as SJS and Aniridia were shown to have a decline in or absence of PAX6 expression.

*Sharma 2012. Human dermal fibroblasts as an alternative feeder layer to 3T3s for the culture of limbal epithelial cells and oral mucosal epithelial cells.

*Lu et al. Human fibroblast cell lines as an alternative to 3T3s for supporting corneal epithelial cell expansion.

1. Tseng SC, Chen SY, Shen YC, Chen WL, Hu FR. Critical appraisal of ex vivo expansion of human limbal epithelial stem cells. Current molecular medicine 10(9), $841-850$ (2010).

2. Utheim TP, Utheim OA, Khan QE, Sehic A. Culture of oral mucosal epithelial cells for the purpose of treating limbal stem cell deficiency. Journal of functional biomaterials 7(1), (2016).

3. Dziasko MA, Armer HE, Levis HJ, Shortt AJ, Tuft S, Daniels JT. Localisation of epithelial cells capable of holoclone formation in vitro and direct interaction with stromal cells in the native human limbal crypt. PloS one 9(4), e94283 (2014).

4. Miyashita H, Shimmura S, Higa K et al. A novel nih/3t3 duplex feeder system to engineer corneal epithelial sheets with enhanced cytokeratin 15-positive progenitor populations. Tissue engineering. Part A 14(7), 1275-1282 (2008).

5. Grueterich M, Espana EM, Tseng SC. Modulation of keratin and connexin expression in limbal epithelium expanded on denuded amniotic membrane with and without a 3t3 fibroblast feeder layer. Investigative ophthalmology \& visual science 44(10), 4230-4236 (2003).

6. Green $\mathrm{H}$. The birth of therapy with cultured cells. BioEssays : news and reviews in molecular, cellular and developmental biology 30(9), 897-903 (2008).

7. Scafetta G, Tricoli E, Siciliano $C$ et al. Suitability of human tenon's fibroblasts as feeder cells for culturing human limbal epithelial stem cells. Stem cell reviews 9(6), 847-857 (2013).

8. Sharma SM, Fuchsluger T, Ahmad S et al. Comparative analysis of human-derived feeder layers with 3 t3 fibroblasts for the ex vivo expansion of human limbal and oral epithelium. Stem cell reviews 8(3), 696-705 (2012).

9. Omoto $\mathrm{M}$, Miyashita $\mathrm{H}$, Shimmura $\mathrm{S}$ et al. The use of human mesenchymal stem cell-derived feeder cells for the cultivation of transplantable epithelial sheets. Investigative ophthalmology \& visual science 50(5), 2109-2115 (2009).

10. Notara M, Haddow DB, Macneil S, Daniels JT. A xenobiotic-free culture system for human limbal epithelial stem cells. Regenerative medicine 2(6), 919-927 (2007). 
11. Lu R, Bian F, Lin J et al. Identification of human fibroblast cell lines as a feeder layer for human corneal epithelial regeneration. PloS one 7(6), e38825 (2012).

12. O'callaghan AR, Daniels JT, Mason C. Effect of sub-atmospheric oxygen on the culture of rabbit limbal epithelial cells. Current eye research 36(8), 691-698 (2011).

13. Pellegrini $G$, Golisano $O$, Paterna P et al. Location and clonal analysis of stem cells and their differentiated progeny in the human ocular surface. The Journal of cell biology 145(4), 769782 (1999).

14. De Paiva CS, Chen Z, Corrales RM, Pflugfelder SC, Li DQ. Abcg2 transporter identifies a population of clonogenic human limbal epithelial cells. Stem cells 23(1), 63-73 (2005).

15. Madhira SL, Vemuganti G, Bhaduri A, Gaddipati S, Sangwan VS, Ghanekar Y. Culture and characterization of oral mucosal epithelial cells on human amniotic membrane for ocular surface reconstruction. Molecular vision 14, 189-196 (2008).

16. Schrader S, Notara M, Tuft SJ, Beaconsfield M, Geerling G, Daniels JT. Simulation of an in vitro niche environment that preserves conjunctival progenitor cells. Regenerative medicine 5(6), 877-889 (2010).

17. Di lorio E, Barbaro V, Ruzza A, Ponzin D, Pellegrini G, De Luca M. Isoforms of deltanp63 and the migration of ocular limbal cells in human corneal regeneration. Proceedings of the National Academy of Sciences of the United States of America 102(27), 9523-9528 (2005).

18. Krishnan S, lyer GK, Krishnakumar S. Culture \& characterisation of limbal epithelial cells \& oral mucosal cells. The Indian journal of medical research 131, 422-428 (2010).

19. Schrader S, O'callaghan AR, Tuft SJ, Beaconsfield M, Geerling G, Daniels JT. Wnt signalling in an in vitro niche model for conjunctival progenitor cells. Journal of tissue engineering and regenerative medicine, (2012).

20. Li W, Chen YT, Hayashida Y et al. Down-regulation of pax6 is associated with abnormal differentiation of corneal epithelial cells in severe ocular surface diseases. The Journal of Pathology 214(1), 114-122 (2008).

21. Hori Y, Nishida K, Yamato M et al. Differential expression of muc16 in human oral mucosal epithelium and cultivated epithelial sheets. Experimental eye research 87(3), 191-196 (2008).

22. Rama P, Matuska S, Paganoni G, Spinelli A, De Luca M, Pellegrini G. Limbal stem-cell therapy and long-term corneal regeneration. The New England journal of medicine 363(2), 147-155 (2010).

23. Kolli S, Ahmad S, Mudhar HS, Meeny A, Lako M, Figueiredo FC. Successful application of ex vivo expanded human autologous oral mucosal epithelium for the treatment of total bilateral limbal stem cell deficiency. Stem cells 32(8), 2135-2146 (2014).

24. Gaddipati S, Muralidhar R, Sangwan VS, Mariappan I, Vemuganti GK, Balasubramanian D. Oral epithelial cells transplanted on to corneal surface tend to adapt to the ocular phenotype. Indian journal of ophthalmology 62(5), 644-648 (2014).

25. Paulsen $\mathrm{F}$, Jager $\mathrm{K}$, Worlitzsch $\mathrm{D}$ et al. Regulation of muc16 by inflammatory mediators in ocular surface epithelial cell lines. Annals of anatomy = Anatomischer Anzeiger : official organ of the Anatomische Gesellschaft 190(1), 59-70 (2008).

26. Hori $Y$, Sugiyama $H$, Soma T, Nishida K. Expression of membrane-associated mucins in cultivated human oral mucosal epithelial cells. Cornea 26(9 Suppl 1), S65-69 (2007).

27. Ahmad S, Stewart R, Yung S et al. Differentiation of human embryonic stem cells into corneal epithelial-like cells by in vitro replication of the corneal epithelial stem cell niche. Stem cells 25(5), 1145-1155 (2007).

28. Blazejewska EA, Schlotzer-Schrehardt U, Zenkel M et al. Corneal limbal microenvironment can induce transdifferentiation of hair follicle stem cells into corneal epithelial-like cells. Stem cells 27(3), 642-652 (2009).

29. Wu J, Du Y, Mann MM, Funderburgh JL, Wagner WR. Corneal stromal stem cells versus corneal fibroblasts in generating structurally appropriate corneal stromal tissue.

Experimental eye research 120, 71-81 (2014). 
30. Lauweryns B, Van Den Oord JJ, De Vos R, Missotten L. A new epithelial cell type in the human cornea. Investigative ophthalmology \& visual science 34(6), 1983-1990 (1993).

31. Wiley L, Sundarraj N, Sun TT, Thoft RA. Regional heterogeneity in human corneal and limbal epithelia: An immunohistochemical evaluation. Investigative ophthalmology \& visual science 32(3), 594-602 (1991).

32. Dua HS, Shanmuganathan VA, Powell-Richards AO, Tighe PJ, Joseph A. Limbal epithelial crypts: A novel anatomical structure and a putative limbal stem cell niche. The British journal of ophthalmology 89(5), 529-532 (2005). 